\title{
论 文
}

\section{北大西洋涛动与西北太平洋热带气旋频数关系的 年代际变化}

\author{
周波涛 ${ }^{(1)(3 *}$, 崔绪 ${ }^{\oplus}$ \\ (1) 国家气候中心, 北京 100081 ; \\ (2) 南京信息工程大学气象灾害预报预警与评估协同创新中心, 南京 210044; \\ (3) 中国科学院大气物理研究所竺可桢-南森国际研究中心, 北京 100029; \\ (4) 国家气象中心, 北京 100081 \\ *E-mail: zhoubt@cma.gov.cn
}

收稿日期: 2013-07-18; 接受日期: 2013-12-19; 网络版发表日期: 2014-04-16

公益性行业(气象)科研专项(编号: GYHY201306026)、国家自然科学基金项目(批准号: 41275078)和国家重点基础研究发展计划项目(编号: 2009CB421407)资助

\begin{abstract}
摘要 基于观测资料分析, 研究了夏季北大西洋涛动与西北太平洋热带气旋频数之间的 关系, 发现两者的联系呈现由弱转强的年代际变化特征. 1948 1977 年, 北大西洋涛动与西 北太平洋热带气旋频数的联系较弱; 但 1980 2009 年, 两者转为显著的正相关, 北大西洋涛 动偏强(弱) 对应西北太平洋热带气旋数偏多(少). 本文进一步从大气环流变化角度, 探讨了 北大西洋涛动与西北太平洋热带气旋频数的关系在前后两个时段出现不同的可能原因. 结 果表明, 在后一时段, 当北大西洋涛动偏强时, 西北太平洋低层为异常气旋型环流, 季风槽 加强, 西太平洋副热带高压偏东, 西北太平洋地区高层大气异常辐散, 低层大气异常辐合, 这些条件均有利于热带气旋的生成和发展, 西北太平洋热带气旋因此偏多. 然而, 在前一时 段, 北大西洋涛动对上述环流系统的影响不明显, 因而减弱了北大西洋涛动与西北太平洋 热带气旋频数的联系. 研究进一步揭示, 与北大西洋涛动异常相关联的波活动通量的变化 可以解释北大西洋涛动与西北太平热带气旋频数联系的这种年代际变化.
\end{abstract}

关键词

热带气旋频数

北大西洋涛动

年代际

波活动通量

大气环流
热带气旋对社会经济发展和生命财产安全影响 很大，造成的灾害也非常严重. 西北太平洋是全球热 带气旋发生频数最高的海域, 因此, 西北太平洋热带 气旋活动尤其受到关注, 有关西北太平洋热带气旋 活动的变化机理和可预测理论研究一直是国际研究 重点.

西北太平洋热带气旋活动(包括气旋生成的个
数、源地、路径和强度)的变化主要取决于影响其生 成发展的大气环流、海温等热力、动力和环境条件 (Gray, 1968; 陈联寿和丁一汇, 1979). 大尺度和行星 尺度大气环流系统也可以通过影响大气辐合辐散、纬 向风垂直切变、海温、西北太平洋季风槽等, 进而影 响西北太平洋热带气旋活动(丁一汇和莱特, 1983; Nakazawa，1988; Liebmann 等，1994; 范可，2007a;

中文引用格式: 周波涛, 崔绚. 2014. 北大西洋涛动与西北太平洋热带气旋频数关系的年代际变化. 中国科学: 地球科学, 44: 1026-1033

英文引用格式: Zhou B T, Cui X. 2014. Interdecadal change of the linkage between the North Atlantic Oscillation and the tropical cyclone frequency over the western North Pacific. Science China: Earth Sciences, doi: 10.1007/s11430-014-4862-Z 
Zhou 和 Cui, 2008). 近些年来, 一些研究探讨了大气 涛动或遥相关型对西北太平洋热带气旋频数的影响. 例如, 王会军等(2007)通过对观测资料和数值模拟结 果的分析, 发现北太平洋涛动(NPO)处于正异常位相 时, 西太平洋区域纬向风垂直切变幅度减小、海表温 度增高, 有利于西太平洋台风生成和发展, 反之亦然. 范可(2007b)还将 NPO 作为预报因子之一, 建立了西 太平洋台风预测模型, 该模型能较合理的反映西太 平洋台风频数的年际变化. 夏季亚洲-太平洋涛动 (APO) 异常变化也对西北太平洋热带气旋活动具有 重要影响. APO 偏强(弱)有(不)利于热带气旋的生成 和发展, 西北太平洋热带气旋因而偏多(少)(周波涛 等, 2008). 同时, APO 的变化还影响西太平洋热带气 旋的活动路径(邹燕和赵平, 2009). 南半球南极涛动 (AAO)信号异常同样影响着西北太平洋热带气旋的 多寡(Ho 等, 2005; 王会军和范可, 2006), 两者之间呈 现显著的反位相变化关系.

上述的这些研究工作揭示出两半球中高纬大气 环流对西北太平洋热带气旋活动具有重要作用. 北 大西洋涛动 $(\mathrm{NAO})$ 是北大西洋地区大气最显著的模 态(Walker 和 Bliss, 1932; Wallace 和 Gutzler, 1981; Rogers, 1984; Hurrell, 1995), 它表征的是亚速尔高压 和冰岛低压之间的尧尧板变化现象. 当亚速尔地区 气压偏高时, 冰岛地区气压偏低; 反之, 当亚速尔地 区气压偏低时, 冰岛地区气压偏高. NAO 模态一年四 季均存在, 并在全球气候系统中发挥着十分重要的 作用. 它不仅直接影响北大西洋及附近地区的气候, 甚至影响整个北半球的气候 (Hurrell, 1995, 1996; Hurrell 和 Van Loon, 1997; Pekarova 和 Pekar, 2007; Archambault 等, 2010).

一些研究阐述了 NAO 变化对东亚区域大气环流 和气候的影响. 如, 冬季 NAO 与东亚冬季风(武炳义 和黄荣辉, 1999)以及我国冬季气温(谭桂容等, 2010) 的联系较为密切. 冬季 NAO 还可影响到后期的亚洲 夏季风环流和降水(Ogi 等, 2004; Sung 等, 2006). 最 近的一些研究发现, 夏季 NAO 同样调制东亚夏季风 环流的变化(Folland 等, 2009; Linderholm 等, 2011). 杨莲梅等(2008)提出, 夏季 NAO 可通过斯堪的纳维 亚半岛中欧西亚和中亚的准静止波传播影响新疆夏 季降水. 此外, 研究还表明, NAO 在 20 世纪 70 年代 末期出现年代际转折(Hurrell, 1995; Jung 等, 2003; Sun 等, 2008), 并造成一些气候系统的关系发生年代
际变化. Chang 等(2001)认为, 20 世纪 70 年代后期印 度夏季风与 ENSO 关系的减弱可能源于 NAO 的年代 际增强. Sun 等 $(2008,2012)$ 发现，随着夏季 NAO 的年 代际变化, 其与东亚气温和降水的关系由弱转强. 鉴 于 $\mathrm{NAO}$ 在气候系统中的重要作用, 那么夏季 NAO变 化对西北太平洋热带气旋活动是否也具有影响? 这 种关系是否存在年代际变化? 如存在, 其中的物理 机制又是什么? 这些问题目前尚不清楚. 因此, 本文 在以上研究的基础上, 重点探讨 NAO 与西北太平洋 热带气旋频数的关系, 并初步分析其中的可能机制.

\section{1 资料}

本文所用的热带气旋资料来自美国联合台风监 测中心(JTWC). 热带气旋按照最大风速标准一般可 分为热带低压、热带风暴和台风. 本研究所讨论的热 带气旋只包括热带风暴和台风. 大气资料为美国国 家环境预报中心/大气研究中心(NCEP/NCAR)的再分 析资料(Kalnay 等, 1996), 水平分辨率为 $2.5^{\circ} \times 2.5^{\circ}$. 分析的变量包括纬向风、经向风和位势高度. NAO 指 数(NAOI)采用 Hurrell(1995)定义的指数, NAOI 数值 越大(小)指示 $\mathrm{NAO}$ 越强(弱). 文中分析所选的时段为 1948 2009 年夏季(6 9 月平均).

\section{2 结果分析}

为揭示夏季 $\mathrm{NAO}$ 与西北太平洋热带气旋频数 (WNPTCF)之间的关系, 我们首先计算了 1948 2009 年时段内夏季 NAOI 与 WNPTCF 的 25 年滑动相关(图 1). 由图 1 可见, NAOI-WNPTCF 的相关具有明显的 年代际变化特征. 20 世纪 70 年代以前, 两者为弱的 负相关, 之后转为正相关并逐渐加强. 在 1980 年后 的每一个 25 年时段内, NAOI-WNPTCF 的正相关都 非常显著(相关系数均通过 $95 \%$ 信度检验), 说明 NAO 同 WNPTCF 的联系在 20 世纪 70 年代末出现年 代际增强. 在随后的讨论中, 分别选取 1948 1977 年 和 1980 2009 年各 30 年作为 NAO 与 WNPTCF 联系 弱和强的研究时段来代表这种年代际变化. 在弱阶 段(1948 1977 年), NAOI-WNPTCF 的相关系数仅为 -0.16 , 相关不明显, 通不过 $90 \%$ 的信度检验; 在强阶 段(1980 2009 年), NAOI-WNPTCF 的相关系数为 0.48 , 相关显著, 通过 $99 \%$ 的信度检验. 


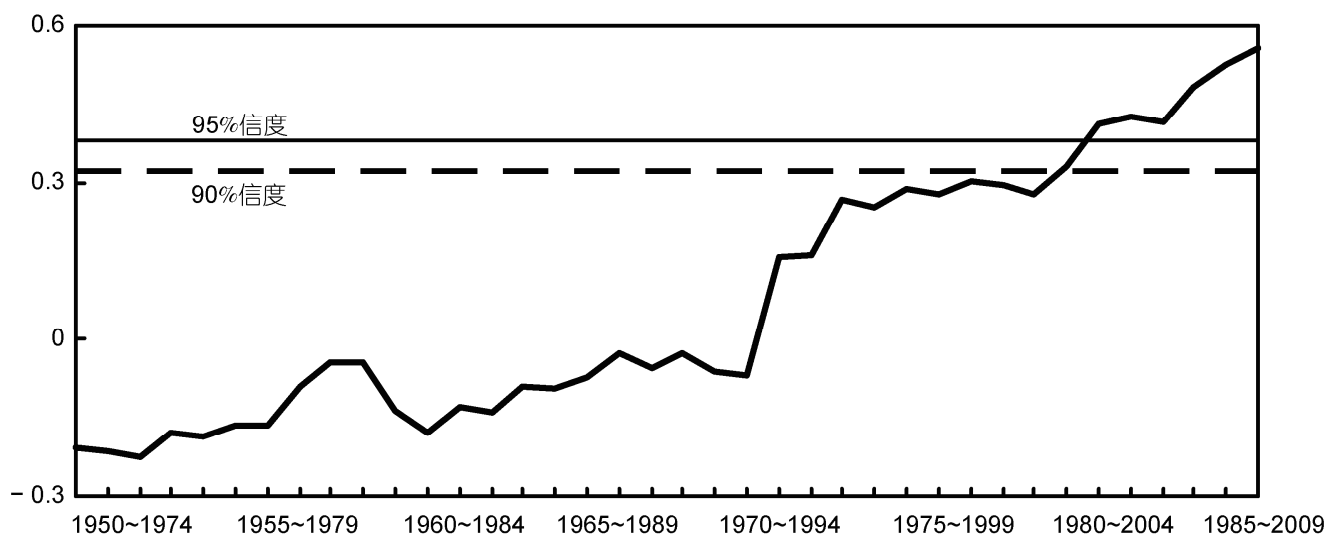

图 1 夏季 NAOI 与 WNPTCF 的 25 年滑动相关

为了解 NAO 与 WNPTCF 关系发生年代际变化 的原因和可能机制, 我们分别从 1948 1977 年和 1980 2009年中, 选取 NAOI 标准值大(小)于 $0.5(-0.5)$ 的年份作为 NAO 正(负)异常年份进行组合分析，探 讨与 NAO 异常变化相关的大气环流背景. 1948 1977 年间正负 NAO 组合分别为 9 年 $(1950,1953,1954$, $1955,1959,1961,1967,1969$ 和 1973 年)和 10 年(1949, 1957，1958，1962，1964，1966，1968，1971，1976 和 1977 年); 1980 2009 年间正负 NAO 组合分别为 9 年 (1981，1988，1989，1990，1992，1994，1996，2005 和 2008 年)和 9 年(1986, 1987, 1993, 1995, 2000, 2001, 2003,2007 和 2009 年).

图 2(a)和(b)分别给出了前后两个时段 $850 \mathrm{hPa}$ 水 平风场的合成差异; 比较两图可以发现, $850 \mathrm{hPa}$ 环 流场存在明显差异. 在前一时段 (1948 1977 年), $\mathrm{NAO}$ 与西北太平洋低空大气环流的联系不明显(图 2(a)). 在后一时段(1980 2009 年), 西北太平洋区域 的环流场呈现偶极型分布, 北侧为异常反气旋型环 流, 南侧为异常气旋型环流. 揭示出 NAO 异常偏强 时, 西太平洋南侧往往出现异常气旋型环流, 季风槽 加强, 这种环流背景有利于西北太平洋热带气旋的 生成和发展( 丁一汇和莱特, 1983; 周波涛和崔绚, 2010), 西北太平洋热带气旋因而增多, 反之亦然.

西太平洋副热带高压的位置变动对热带气旋频 数具有显著影响(丁一汇和莱特, 1983; Ho 等, 2004). 图 3 为 $\mathrm{NAO}$ 正负位相年份对应的 $500 \mathrm{hPa}$ 位势高度 分布. 可见, 在前一时段(图 3(a)), 西太平洋副热带 高压主体位置在强、弱 NAO 年份的变化不大. 但在 后一时段(图 3(b)), 与弱 $\mathrm{NAO}$ 年相比, 强 NAO 年所
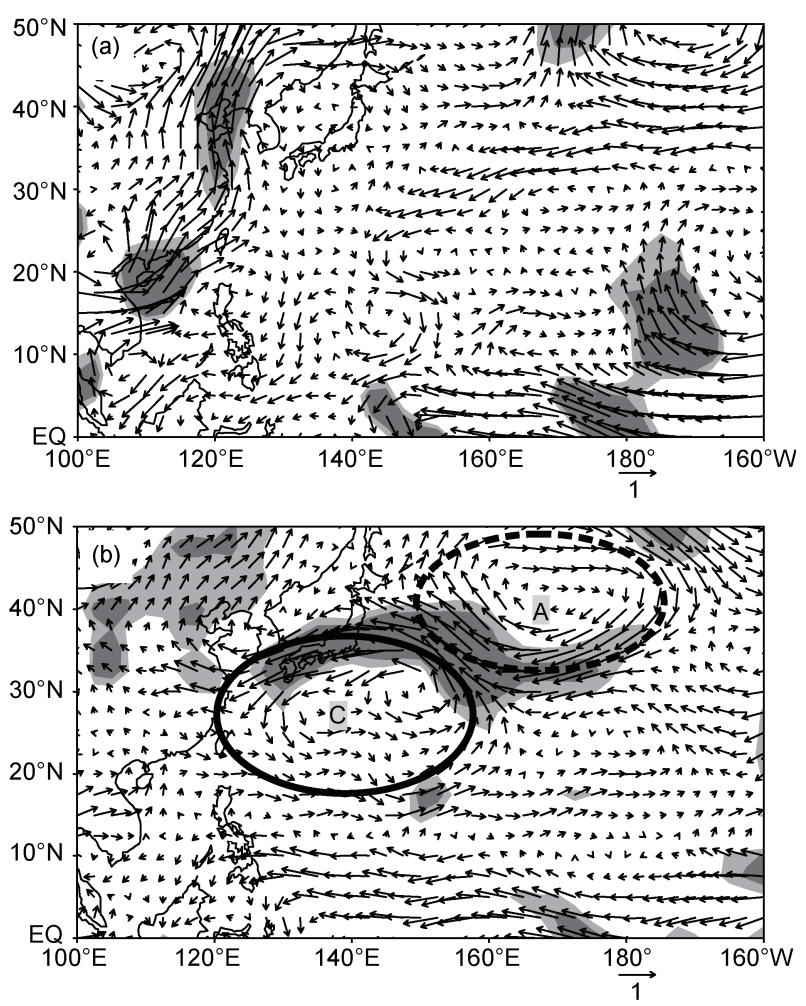

图 2 强弱 $\mathrm{NAO}$ 年 $850 \mathrm{hPa}$ 水平风场合成差异

(a) 1948 1977 年; (b) 1980 2009 年. A, C 分别表示反气旋型环流和 气旋型环流, 深(浅)阴影区表示通过 $95 \%(90 \%)$ 信度. 单位: $\mathrm{m} \mathrm{s}^{-1}$

对应的西太平洋副热带高压的主体位置偏东. $5880 \mathrm{~m}$ 特征线在 NAO 正位相年较 NAO 负位相年东移了约 8 个经度. 因此, 夏季 NAO 偏强时, 西太平洋副热带 高压主体位置偏东, 有利于西北太平洋热带气旋的 生成和发展. 反过来, 当夏季 NAO 偏弱时, 西太平洋 副热带高压主体位置偏偏西, 不利于西北太平洋热带 

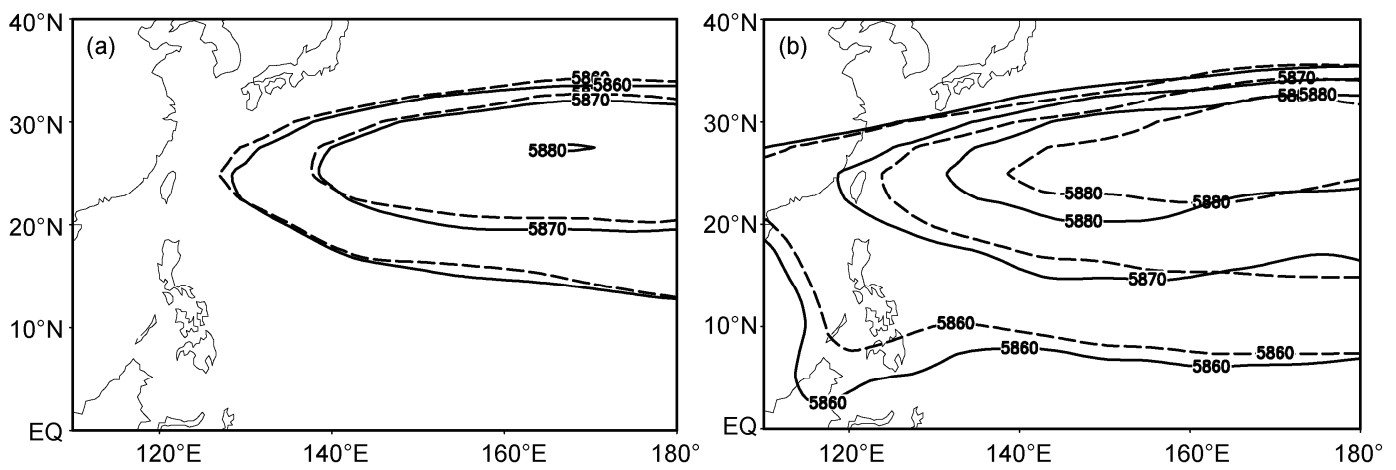

图 3 强(虚线)弱(实线)NAO 年 $\mathbf{5 0 0} \mathrm{hPa}$ 位势高度

(a) 1948 1977 年; (b) 1980 2009 年. 单位: $m$

气旋的生成和发展. 这再次印证了 NAOI-WNPTCF 的 正相关关系.

对流层高低层散度场的相互配置是热带气旋发 生发展的一个重要因素. 图 4 为强、弱 NAO 年 925 $\mathrm{hPa}$ 和 $150 \mathrm{hPa}$ 散度合成差值的空间分布. 在 1980 2009 年(图 4(c)和(d)), 散度场差值分布特征表现为: 低层 $10^{\circ} \sim 30^{\circ} \mathrm{N}$ 纬度带内为负值, 大气异常辐合; 高 层基本上为正值, 大气异常辐散. 这种上下配置作用 将会导致西北太平洋热带气旋主要源地出现强烈的 上升运动，有利于西北太平洋热带气旋的生成发展. 但是, 在前一时段(图 4(a)和(b)), 高低层散度场的这 种配置在西北太平洋热带气旋主要源地并不太显著, 这也就削弱了 $\mathrm{NAO}$ 与西北太平洋热带气旋频数之间 的联系.
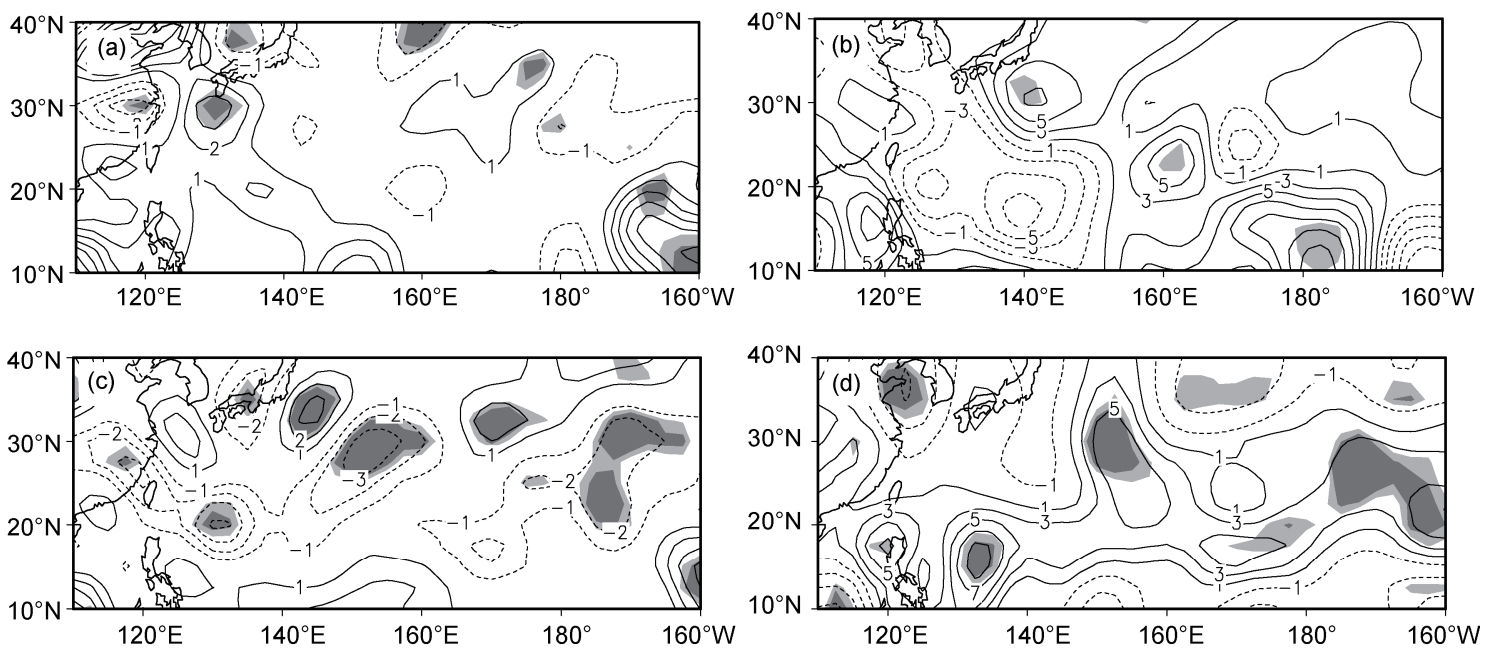

图 4 强弱 $\mathrm{NAO}$ 年对应的 $925 \mathrm{hPa}((\mathrm{a}),(\mathrm{c}))$ 和 $150 \mathrm{hPa}((\mathrm{b}),(\mathrm{d}))$ 散度合成差值
以上分析表明，在 1980 2009 年, NAO 可以通过 影响西北太平洋区域的大气环流，进而与西北太平 洋热带气旋频数变化建立联系. 强 NAO 对应季风槽 加强, 西太平洋副热带高压东移以及高层大气异常 辐散和低层大气异常辐合, 这些为西北太平洋热带 气旋的发生发展提供了有利的环流背景. 然而, 在 1948 1977 年间, NAO 与上述环流的关系不密切, NAO 和 WNPTCF 的联系因而不明显.

为何 $\mathrm{NAO}$ 与西北太平洋地区大气环流的联系在 前后两时段会有所不同? NAO 信号是如何影响西北 太平洋区域的大气环流? 已有研究揭示, 副热带西 风急流作为 Rossby 波传播的波导，可以将 NAO 信号 以准静止波波列形式向下游传播(Hoskins 和 Ambrizzi, 1993; Branstator, 2002; Watanabe, 2004). 为了揭示与 
NAO 相联系的遥相关波列传播, 我们分别计算了两 个时段内 NAOI 与 $200 \mathrm{hPa}$ 经向风的回归分布. 对流 层上层经向风可以较好地刻画纬向遥相关. 从图 5(b) 可以清楚地看到, 沿着副热带西风急流区出现明显 的南北风交替波列, 这支波列一直延伸到北太平洋 地区. 这说明在西风急流波导的作用下, NAO 信号可 以向西北太平洋区域传播, 进而影响局地大气环流. 与此不同, 在 1948 1977(图 5(a)), 波列的传播仅传至 亚洲西部, 东亚西风急流没有捕获到明显的波列. 这 种差异可能与后一时期东亚西风急流增强有关, 西 风急流作为波导可以影响波射线路径(Branstator, 2002; Watanabe, 2004; Sung 等, 2006). Wu 等(2009, 2012)研究发现, 春季 NAO 同样可通过副热带急流的 波导作用将 Rossby 波列从大西洋传到西太平洋, 进 而影响东亚夏季风. 他们基于 $500 \mathrm{hPa}$ 高度层和不同 的分析时段指出, 相比于 1958 1984 年, 1985 2008 年欧亚中部急流宽而弱, 但有 Rossby 波列向下游传 播, 反映副热带急流范围可能也影响波列的传播.

波活动通量是诊断波流相互作用和波动传播的
有效方法. 为此, 我们利用与 NAOI 回归后的位势高 度异常场，进一步计算了同 NAO 有关的波活动通量， 以此探讨准静止波的传播. 水平波活动通量计算公 式(Takaya 和 Nakamura, 2001)为

$$
W=\frac{p}{2|\mathbf{U}|}\left[\begin{array}{l}
U\left(\psi_{x}^{\prime 2}-\psi^{\prime} \psi_{x x}^{\prime}\right)+V\left(\psi_{x}^{\prime} \psi_{y}^{\prime}-\psi^{\prime} \psi_{x y}^{\prime}\right) \\
U\left(\psi_{x}^{\prime} \psi_{y}^{\prime}-\psi^{\prime} \psi_{x y}^{\prime}\right)+V\left(\psi_{y}^{\prime 2}-\psi^{\prime} \psi_{y y}^{\prime}\right)
\end{array}\right] .
$$

式中, $\psi^{\prime}$ 为扰动流函数, $U, V$ 分别为基本气流的纬向 和经向分量, $|\mathbf{U}|$ 为水平风速, $p$ 为气压除以 $1000 \mathrm{hPa}$.

图 6(a)和(b)分别给出了 1948 1977 年和 1980 2009 年与 $\mathrm{NAO}$ 相联系的 $200 \mathrm{hPa}$ 波活动通量的变化. 比较两图可以发现, 前后两个时段的波通量分布存 在明显差异. 在前一时段里(图 6(a)), 北大西洋区域 的波通量较强, 在流函数场上表现为南北向的偶极 型. 其他区域的波通量相对较弱. 这意味着 NAO 信 号局限于大西洋地区, 没有向下游传播. 在后一时段 (图 6(b)), 大西洋地区仍为波活动通量大值区，但在流 函数场上表现为三极型. 此外, 波活动通量明显向下 游扩展, 在亚洲、北太平洋区域也出现波通量大值区.
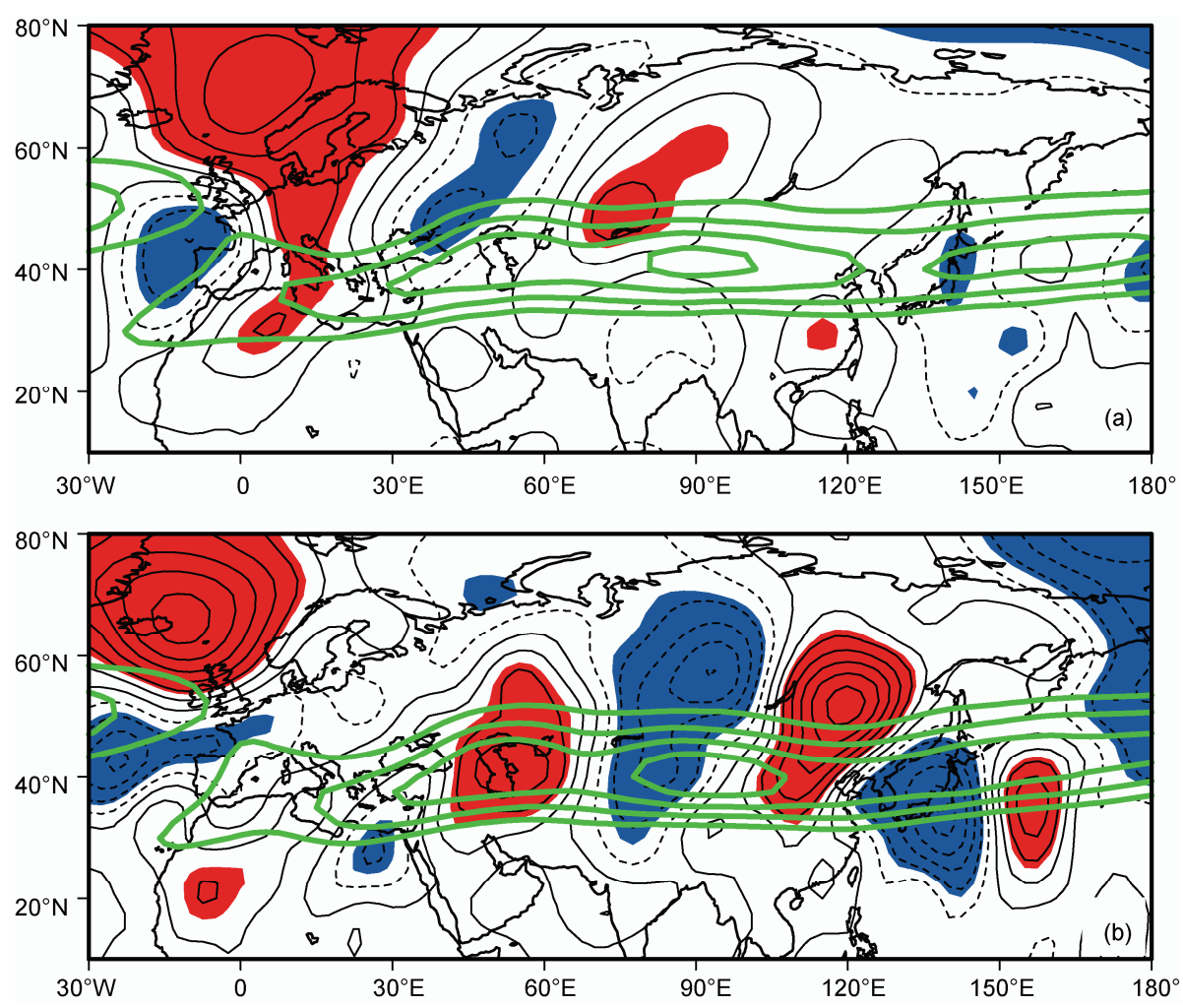

图 $5200 \mathrm{hPa}$ 经向风与 NAOI 的回归分布

(a) 1948 1977 年; (b) 1980 2009 年. 绿线, 叠加了 $200 \mathrm{hPa}$ 纬向风气候态. 单位: $\mathrm{m} \mathrm{s}^{-1}$ 

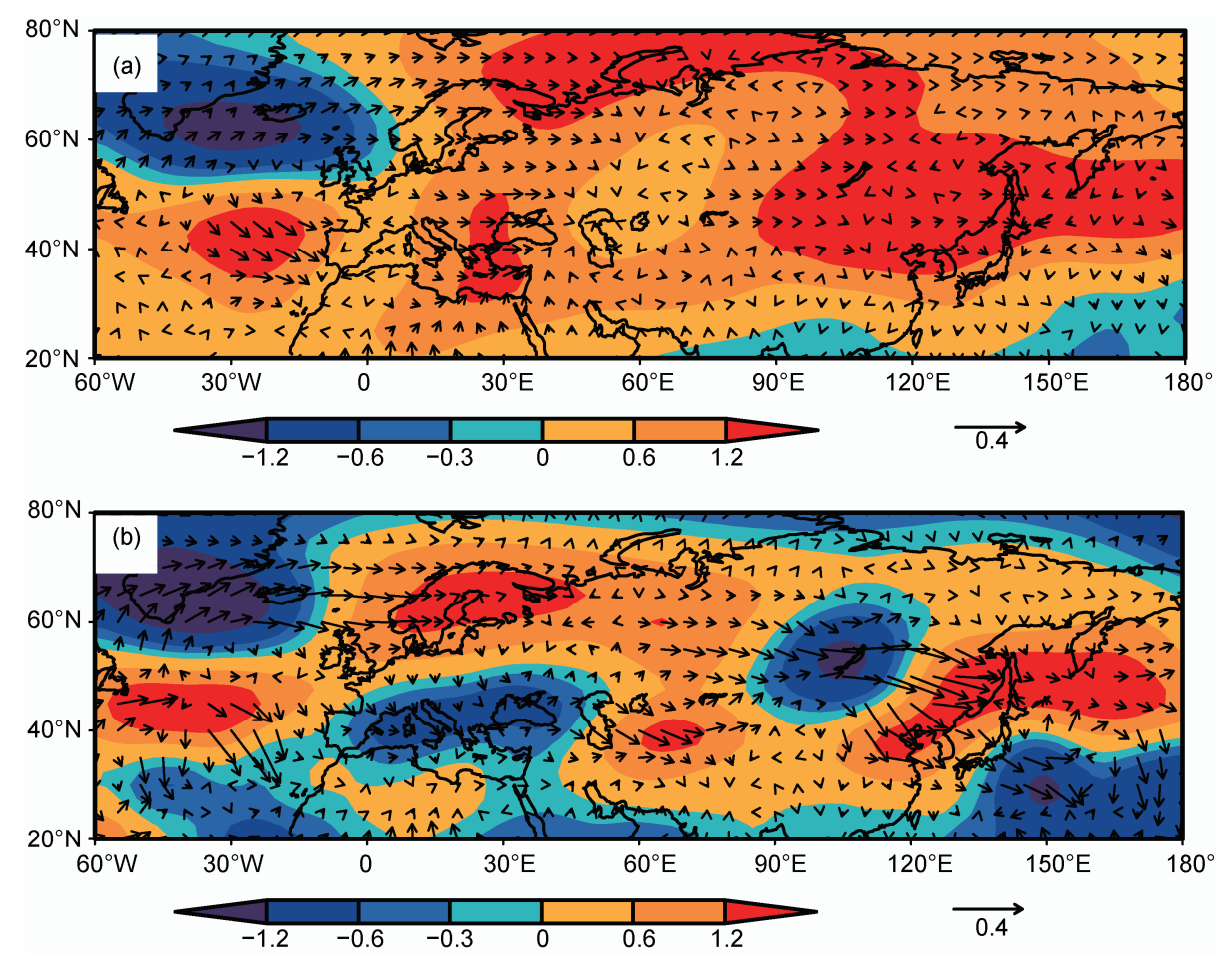

图 6 与 NAO 相联系的 $200 \mathrm{hPa}$ 水平波活动通量与流函数

(a) 1948 1977 年; (b) 1980 2009 年. 矢量, 单位: $\mathrm{m}^{2} \mathrm{~s}^{-2}$; 阴影, 单位: $10^{6} \mathrm{~m}^{2} \mathrm{~s}^{-1}$

而且, 副热带地区存在明显的纬向波列, 这个波列将 $\mathrm{NAO}$ 信号从大西洋向东传播到西北太平洋区域, 从 而影响到该地的大气环流. 因此, 波活动通量异常在 $\mathrm{NAO}$ 同西北太平洋大气环流的联系中起着重要的桥 梁作用. 两个时段内与 $\mathrm{NAO}$ 变化相关联的波活动通 量的不同造成夏季 NAO 与 WNPTCF 的关系出现年 代际变化.

\section{3 讨论与结论}

本文研究了夏季 NAO 与 WNPTCF 之间的关系, 发现 NAO 与 WNPTCF 的联系在 20 世纪 70 年代末 发生了一次明显的年代际转变. 之前, NAO 和 WNPTCF 的关系淡漠, 两者间的相关系数仅为 -0.16 ; 之后, NAO 与 WNPTCF 的对应关系明显增强, 呈现 显著的正相关，相关系数达到 0.48 (通过 $99 \%$ 的信度 检验). 亦即, 当 NAO 异常偏强时, 西北太平洋热带 气旋往往偏多; 反之, 当 NAO 异常偏弱时, 西北太 平洋热带气旋往往偏少.

本文进一步从大气环流角度探讨了 $\mathrm{NAO}$ 与
WNPTCF 关系发生年代际变化的可能原因和物理机 制. 结果表明, 1980 2009 年, 当 NAO 位于正位相时, 西北太平洋低层出现异常气旋型环流, 季风槽加强, 西太平洋副热带高压主体位置偏东; 西太平洋地区 高层大气异常辐散, 低层大气异常辐合, 利于对流活 动发展, 这些均为热带气旋的发生发展提供了有利 的环流背景, 西北太平洋热带气旋因而偏多. NAO 位 于负位相时情况则相反. 但是, 与此不同, NAO 与上 述大气环流条件的联系在 1948 1977 年间不密切，从 而削弱了 NAO 与 WNPTCF 之间的联系. 研究还揭示, 波活动通量的加强和东移是造成后一时段 NAO 与 WNPTCF 关系加强的可能原因. 在前一段时间 (1948 1977 年), 与 NAO 有关的波通量异常在北大西 洋很强, 但在其他区域相对较弱, 因此, NAO 信号不 能向下游传播，从而无法影响西北太平洋区域的大 气环流. 但在后一段时间(1980 2009 年), 波活动通 量加强并明显向下游扩展, NAO 信号通过副热带地 区的纬向波列东传至西北太平洋区域，从而能影响 到该地的大气环流, 进而引起西北太平洋热带气旋 的多寡变化. 
本文只是从波活动通量角度, 初步解释了夏季 $\mathrm{NAO}$ 与西北太平洋热带气旋频数关系的年代际变化, 这只是其中的一种物理机制, 还可能有其他途径联 系着两者关系的变化. 已有的研究揭示, 位于急流入 口附近的地中海地区高层大气异常辐散可以激发 Rossby 波列, 在 NAO 信号向下游传播中起着重要作 用. NAO 信号和地中海高层大气异常辐合被看作是 联接 NAO 和东亚气候的关键物理过程之一 (Watanabe, 2004). 此外, 急流区是大气的强斜压区, 在准定常急流下游稍北处存在高频瞬变扰动, 这种 淤动的带通瞬变方差峰值区通常称为太平洋风暴轴 和大西洋风暴轴. 北太平洋风暴轴与急流变化密切 相关, 风暴轴的向极移动伴随着西风急流的向极地 移动(Lau等, 2004), 而且风暴轴位置的南北摆动还影 响 NAO 位相的变化(Franzke 等, 2004). 再者, 太平 洋-北美遥相关型(PNA)可以调整北半球行星波振幅 和风暴轴路径以及东亚西风急流的位置与强度, 并 同 NAO 呈负位相变化关系(Song 等, 2009). 那么, 太
平洋风暴轴和 PNA 的变化对 $\mathrm{NAO}$ 与西北太平洋热 带气旋频数关系的年代际变化是否具有贡献? ENSO 同样对 NAO 变化具有影响(Trenberth 等, 1998; PozoVázquez 等, 2001; Mokhov 和 Smirnov, 2006). 如 Pozo-Vázquez 等(2001)的研究表明, 北大西洋区域在 ENSO 冷位相期间呈现类 NAO 正位相环流型. ENSO 对西北太平洋热带气旋活动的作用已被不少研究所 揭示. ENSO 可以通过影响纬向风垂直切变幅度、区 域大气辐合辐散、Walker 环流、季风槽活动等进而 影响热带气旋的频次、强度和位置(Chan, 2000; Chia 和 Ropelewski, 2002; Wang 和 Chan, 2002; Camargo 和 Sobel, 2005). 而且, ENSO 事件也具有明显的年代 际变化特征(Wang，1995; Wang 等，2009). 那么, ENSO 在 NAO 与西北太平洋热带气旋频数年代际 关系变化中又起着何种作用? 这些因子的各自贡献 有多大？这些问题都还有待深入探讨，需要今后作 更多的定量诊断以及利用先进的气候模式作进一步 研究.

\section{参考文献}

陈联寿, 丁一汇. 1979. 西太平洋台风概论. 北京: 科学出版社. 1-10

丁一汇，莱特. 1983. 影响西太平洋台风形成的大尺度环流条件. 海洋学报, 5: 561-574

范可. 2007a. 北太平洋海冰, 一个西北太平洋台风生成频次的预测因子? 中国科学 D 辑: 地球科学, 37: 851-856

范可. 2007b. 西北太平洋台风生成频次的新预测因子和新预测模型. 中国科学 D 辑：地球科学, 37: 1260-1266

谭桂容, 陈海山, 孙照渤, 等. 2010. 2008 年 1 月中国低温与北大西洋涛动和平流层异常活动的联系. 大气科学, 34: 175-183

王会军, 孙建奇, 范可. 2007. 北太平洋涛动与台风和揕风频次的关系研究. 中国科学 D 辑: 地球科学, 37: 966-973

王会军, 范可. 2006. 西北太平洋台风生成频次与南极涛动的关系. 科学通报, 51:2910-2914

武炳义, 黄荣辉. 1999. 冬季北大西洋涛动极端异常变化与东亚冬季风. 大气科学, 23: 641-651

杨莲梅, 张庆云. 2008. 北大西洋涛动对新疆夏季降水异常的影响. 大气科学, 32: 1187-1196

周波涛, 崔绚, 赵平. 2008. 亚洲-太平洋涛动与西北太平洋热带气旋频数的关系. 中国科学 D 辑：地球科学, 38: 118-123

周波涛, 崔绚. 2010. 澳大利亚东侧海温: 西北太平洋热带气旋生成频数的预测信号? 科学通报, 55: 3053-3059

邹燕, 赵平. 2009. 夏季亚洲-太平洋涛动与中国近海热带气旋活动的关系. 气象学报, 67: 708-715

Archambault H M, Keyser D, Bosart L F. 2010. Relationships between large-scale regime transitions and major cool-season precipitation events in the Northeastern United States. Mon Weather Rev, 138: 3454-3473

Branstator G. 2002. Circumglobal teleconnections, the jet stream waveguide, and the North Atlantic Oscillation. J Clim, 15: 1893-1910 Camargo S J, Sobel A H. 2005. Western North Pacific tropical cyclone intensity and ENSO. J Clim, 18: 2996-3006

Chan J C L. 2000. Tropical cyclone activity over the western North Pacific associated with El Niño and La Niña events. J Clim, 13: 2960-2972

Chang C P, Harr P, Ju J. 2001. Possible roles of Atlantic circulations on the weakening Indian monsoon rainfall-ENSO relationship. J Clim, 14: 2376-2380

Chia H H, Ropelewski C F. 2002. The interannual variability in the genesis location of tropical cyclones in the Northwest Pacific. J Clim, 15: 2934-2944 Folland C K, Knight J, Linderholm H W, et al. 2009. The Summer North Atlantic Oscillation: Past, present, and future. J Clim, 22: 1082-1103

Franzke C, Lee S, Feldstein S B. 2004. Is the North Atlantic Oscillation a breaking wave? J Atmos Sci, 61: 145-160

Gray W M. 1968. Global view of the origin of tropical disturbances and storms. Mon Weather Rev, 96: 669-700

Ho C H, Baik J J, Kim J H, et al. 2004. Interdecadal changes in summertime typhoon tracks. J Clim, 17: 1767-1776

Ho C H, Kim J H, Kim H S, et al. 2005. Possible influence of the Antarctic Oscillation on tropical cyclone activity in the western North Pacific. J 
Geophys Res, 110: D19104, doi: 10.1029/2005JD005766

Hoskins B J, Ambrizzi T. 1993. Rossby wave propagation on a realistic longitudinally varying flow. J Atmos Sci, 50: 1661-1671

Hurrell J W. 1995. Decadal trends in the North Atlantic Oscillation: Regional temperatures and precipitation. Science, 269: 676-679

Hurrell J W. 1996. Influence of variations in extratropical wintertime teleconnections on Northern Hemisphere temperature. Geophys Res Lett, 23: $665-668$

Hurrell J W, van Loon H. 1997. Decadal variations associated with the North Atlantic Oscillation. Clim Change, 36: 301-326

Jung T, Hilmer M, Ruprecht E, et al. 2003. Characteristics of the recent eastward shift of interannual NAO variability. J Clim, 16: 3371-3382

Kalnay E, Kanamistu M, Kistler R, et al. 1996. NCEP/NCAR 40-year reanalysis project. Bull Amer Meteorol Soc, 77: 437-471

Lau K M, Lee J Y, Kim K M, et al. 2004. The North Pacific as a regulator of summertime climate over Eurasia and North America. J Clim, 17: 819-833

Liebmann B, Hendon H H, Glick J D. 1994. The relationship between tropical cyclones of the western Pacific and Indian Oceans and the Madden-Julian Oscillation. J Meteorol Soc Jpn, 72: 401-411

Linderholm H W, Ou T, Jeong J H, et al. 2011. Interannual teleconnections between the summer North Atlantic Oscillation and the East Asian summer monsoon. J Geophys Res, 116: D13107, doi:10.1029/2010JD015235

Mokhov I I, Smirnov D A. 2006. El Nino-Southern Oscillation drives North Atlantic Oscillation as revealed with nonlinear techniques from climatic indices. Geophys Res Lett, 33: L03708, doi: 10.1029/2005GL024557

Nakazawa T. 1988. Tropical super clusters within intraseasonal variations over the western Pacific. J Meteorol Soc Jpn, 66: 823-839

Ogi M, Tachibana Y, Yamazaki K. 2004. The connectivity of the winter North Atlantic Oscillation and the summer Okhotsk high. J Meteorol Soc Jpn, 82: 905-913

Pekarova P, Pekar J. 2007. Teleconnection of interannual streamflow fluctuation in Slovakia with Arctic Oscillation, North Atlantic Oscillation, Southern Oscillation, and Quasi-biennial Oscillation phenomena. Adv Atmos Sci, 24: 655-663

Pozo-Vázquez D, Esteban-Parra M J, Rodrigo F S, et al. 2001. The association between ENSO and winter atmospheric circulation and temperature in the North Atlantic region. J Clim, 16: 3408-3420

Rogers J C. 1984. The association between the North Atlantic Oscillation and the Southern Oscillation in the Northern Hemisphere. Mon Weather Rev, 112: 1999-2015

Song J, Li C Y, Zhou W, et al. 2009. The linkage between the Pacific-North American teleconnection pattern and the North Atlantic Oscillation. Adv Atmos Sci, 26: 229-239

Sun J Q, Wang H J, Yuan W. 2008. Decadal variations of the relationship between the summer North Atlantic Oscillation and middle East Asian air temperature. J Geophys Res, 113: D15107, doi: 10.1029/2007JD009626

Sun J Q, Wang H J. 2012. Changes of the connection between the summer North Atlantic Oscillation and the East Asian summer rainfall. J Geophys Res, 117: D08110, doi: 10.1029/2012JD017482

Sung M K, Kwon W T, Baek H J. 2006. A possible impact of the North Atlantic Oscillation on the East Asian summer monsoon precipitation. Geophys Res Lett, 33: L21713, doi: 10.1029/2006GL027253

Takaya K, Nakamura H. 2001. A Formulation of a phase-independent wave-activity flux for stationary and migratory quasigeostrophic eddies on a zonally varying basic flow. J Atmos Sci, 58: 608-627

Trenberth K E, Branstator G W, Karoly D, et al. 1998. Progress during TOGA in understanding and modeling global teleconnections associated with tropical sea surface temperatures. J Geophys Res, 103: 14291-14324

Walker G T, Bliss E W. 1932. World Weather. V Mem R Meteorol Soc, 4: 53-84

Wallace J M, Gutzler D S. 1981. Teleconnections in the geopotential height field during the Northern Hemisphere winter. Mon Weather Rev, 109: 784-812

Wang B. 1995. Interdecadal changes in El Nino onset in the last four decades. J Clim, 8: 267-285

Wang B, Chan J C L. 2002. How strong ENSO events affect tropical storm activity over the western North Pacific. J Clim, 15: 1643-1658

Wang X, Wang D X, Zhou W. 2009. Decadal variability of twentieth-century El Niño and La Niña occurrence from observations and IPCC AR4 coupled models. Geophys Res Lett, 36: L11701, doi: 10.1029/2009GL037929

Watanabe M. 2004. Asian jet waveguide and a downstream extension of the North Atlantic Oscillation. J Clim, 17: 4674-4691

Wu Z W, Wang B, Li J P, et al. 2009. An empirical seasonal prediction model of the East Asian summer monsoon using ENSO and NAO. J Geophys Res, 114: D18120, doi: 10.1029/2009JD011733

Wu Z W, Li J P, Jiang Z H, et al. 2012. Possible effects of the North Atlantic Oscillation on the strengthening relationship between the East Asian Summer monsoon and ENSO. Int J Climatol, 32: 794-800

Zhou B T, Cui X. 2008. Hadley circulation signal in the tropical cyclone frequency over the western North Pacific. J Geophys Res, 113: D16107, doi: 10.1029/2007JD009156 